\title{
Cardiac Complications Associated with COVID-19, MIS-C, and mRNA COVID-19 Vaccination
}

\author{
Stephanie E. Chin ${ }^{1,3,4} \cdot$ Sejal M. Bhavsar ${ }^{2,3} \cdot$ Andrew Corson ${ }^{1} \cdot$ Zelda J. Ghersin $^{1,3} \cdot$ Hannah S. Kim ${ }^{2,3}$
}

Received: 16 December 2021 / Accepted: 12 February 2022 / Published online: 8 March 2022

(c) The Author(s), under exclusive licence to Springer Science+Business Media, LLC, part of Springer Nature 2022

\begin{abstract}
The COVID-19 vaccine is now approved for individuals greater than 5 years of age, but vaccination rates remain lower than expected in the pediatric age group. Misinformation and widespread reporting of vaccine-related myocarditis are contributing to vaccine hesitancy. When compared to severe cardiac complications that are associated with COVID-19, vaccine-related myocarditis has a milder presentation, is easily treated, and has a good prognosis. Acute COVID-19 has been associated with higher rates of myocarditis and myocardial injury. Multisystem inflammatory syndrome in children occurs weeks after initial infection with SARS-CoV-2 and can be associated with severe cardiovascular complications and death. Cardiac complications associated with acute COVID-19 and MIS-C are more severe and occur more frequently than myocarditis after mRNA COVID-19 vaccination. Furthermore, some of the academic and social disruptions caused by the pandemic expect to be eased by widespread vaccination. For all these reasons, COVID-19 vaccination is strongly recommended for all eligible age groups.
\end{abstract}

Keywords COVID-19 $\cdot$ Myocarditis · Vaccination

\begin{tabular}{|c|c|}
\hline \multicolumn{2}{|c|}{ Abbreviations } \\
\hline ACE2 & Angiotensin-converting enzyme 2 \\
\hline BNP & Brain natriuretic peptide \\
\hline $\mathrm{CDC}$ & $\begin{array}{l}\text { Centers for Disease Control and } \\
\text { Prevention }\end{array}$ \\
\hline cMRI & Cardiac magnetic resonance imaging \\
\hline COVID-19 & Coronavirus disease 2019 \\
\hline ECMO & Extracorporeal membrane oxygenation \\
\hline EKG & Electrocardiogram \\
\hline ICU & Intensive care unit \\
\hline IFN & Interferon \\
\hline $\mathrm{IL}$ & Interleukin \\
\hline IVIG & Intravenous immune globulin \\
\hline MERS-CoV & $\begin{array}{l}\text { Middle East respiratory syndrome } \\
\text { coronavirus }\end{array}$ \\
\hline
\end{tabular}

Stephanie E. Chin

schin@alpertzalescastro.com

1 K. Hovnanian Children's Hospital at Jersey Shore University Medical Center, Neptune, NJ, USA

2 Joseph M. Sanzari Children's Hospital at Hackensack University Medical Center, Hackensack, NJ, USA

3 Hackensack Meridian School of Medicine, Nutley, NJ, USA

4 Alpert Zales Castro Pediatric Cardiology, 1623 Route 88, Brick, NJ 08724, USA

\author{
mRNA Messenger ribonucleic acid \\ SARS-CoV-2 Severe acute respiratory syndrome corona- \\ virus 2 \\ TNF Tumor necrosis factor \\ VAERS Vaccine Adverse Event Reporting System
}

\section{Introduction}

There has been vaccine hesitancy among families of young children who are now eligible to receive the coronavirus disease 2019 (COVID-19) vaccine. This hesitancy remains despite clear recommendations from the Centers for Disease Control and Prevention (CDC) that everyone five years and older receive the COVID-19 vaccine [1]. As of December 1, $2021,15 \%$ of 5-11-year olds have received at least one dose of the COVID-19 vaccine and 60\% of 12-17-year olds have received at least one dose of the COVID-19 vaccine, with wide variation across states [2]. Misinformation and widespread reporting of vaccine-related myocarditis are likely contributing to this hesitancy, although this adverse reaction is rare, easily treated, and has a good prognosis. While it is known that older individuals are much more likely to become severely ill or require mechanical ventilation from COVID-19, with greater than $80 \%$ of deaths related to COVID-19 occurring in the 65 and older population [1], it 
is important to remember that although rare, children who contract severe acute respiratory syndrome coronavirus 2 (SARS-CoV-2) are at risk for severe outcomes, including post-infective myocarditis, multisystem inflammatory syndrome in children (MIS-C), and even death. As more of the older population gets vaccinated, adolescents and young adults aged 12-29 years represent an increasing proportion of COVID-19 cases [3]. Although most children who contract SARS-CoV-2 are asymptomatic or have mild illness, the CDC has reported 2095 deaths due to COVID-19 among those aged 5 to 24 years as of November 24, 2021 [4]. This paper serves to review cardiac complications associated with acute COVID-19 infection, MIS-C, and vaccine-related myocarditis.

\section{SARS-CoV-2 and Myocarditis}

Viral infections such as adenovirus and enterovirus are recognized as common causes of myocarditis, manifesting as localized or global myocardial inflammation, tissue necrosis, and ventricular dysfunction from direct viral invasion [5]. Myocarditis has also been identified as a complication of severe coronaviruses, including SARS-CoV and Middle East respiratory syndrome coronavirus (MERS-CoV) [6, 7]. The exact mechanism of SARS-CoV-2 infection leading to myocarditis is unknown. While some investigators believe SARS-CoV-2 enters the cardiomyocyte through an angiotensin-converting enzyme 2 (ACE2)-mediated entry and directly damages the myocardium, others believe that myocarditis may occur from host inflammatory cell response. Emerging data indicate that a host immune response with excessive activation of innate immune pathways along with proinflammatory cytokine surge, deregulated thromboinflammation, thrombotic microangiopathy, and endothelial dysfunction may play a role in pathogenesis of cardiac injury related to COVID-19 [8].

While initial reports of SARS-CoV-2 infection focused largely on respiratory complications, cardiovascular complications have also been reported and are contributing greatly to the morbidity and mortality in adult patients. The cardiovascular complications include myocardial injury, myocarditis, acute myocardial infarction, heart failure, dysrhythmias, and venous thromboembolic events $[9,10]$. Pediatric cases of fulminant myocarditis [11-13] and arrhythmias have been reported $[14,15]$. These pediatric patients have not had any significant underlying health conditions and tested positive for SARS-CoV-2 via polymerase chain reaction. Cardiac complications have been reported in neonates as young as nine days of life [15]. In collegiate athletes recovering from acute COVID-19, up to $3 \%$ of previously healthy athletes had cardiac magnetic resonance imaging (cMRI) abnormalities consistent with myocarditis [16].
Using a large hospital-based administrative database, a CDC study revealed that during March 2020-January 2021, individuals with COVID-19 had 15.7 times the risk for myocarditis compared with individuals who did not have COVID-19 and risk varied by sex and age. The myocarditis risk is 37 times higher for infected children under 16 years and seven times higher for infected people aged 16-39 years compared to their uninfected peers. The risk difference for myocarditis was increased in males compared to females [17]. The study investigators report that some of these pediatric myocarditis cases may be due to cases of MIS-C, which is further described below.

\section{COVID-19 and MIS-C}

Over ten and a half million children have been infected with SARS-CoV-2 in the USA [18], with less than $1 \%$ developing a post-infectious inflammatory syndrome known as multisystem inflammatory syndrome in children (MIS-C) [19]. MIS-C generally occurs three to six weeks after infection with SARS-CoV-2 [20]. Per the CDC, as of January 3, 2022, at least 6,431 children have met diagnostic criteria for MISC, with 55 confirmed deaths in the USA alone [19]. A large international network cohort study on children aged 0 to 19 years showed that MIS-C affected 0.5 to $3.1 \%$ of children and up to $0.9-7.6 \%$ of hospitalized children with COVID-19 [21]. As the name suggests, this post-infectious syndrome affects multiple organ systems, including cardiovascular. Although the percentage of children who develop MIS-C after SARS-CoV-2 infection is small, as the pandemic continues we can anticipate increased incidence.

While the long-lasting cardiovascular effects of MIS-C are still unknown, patients may suffer both acute and subacute complications from disease and cardiovascular manifestations range from mild to severe. MIS-C can resemble Kawasaki disease as they both have similar laboratory and clinical features. In contrast, however, MIS-C disproportionately affects African American and Hispanic patients, occurs at an older age (mean age of nine years old), and appears to have a higher incidence of myocarditis, elevated troponins, ventricular dysfunction, and vasopressor requirements [22]. It has been reported that between 50 and $70 \%$ of patients with MIS-C have myocarditis [23, 24] and in a study by Son et al. evaluating 518 hospitalized pediatric patients diagnosed with MIS-C, 47\% required vasopressor support, $41 \%$ had depressed left ventricular systolic dysfunction, $12 \%$ developed coronary artery aneurysm, and 3\% required extracorporeal membrane oxygenation (ECMO) [25]. Furthermore, in a case series evaluating 20 patients with MIS-C and cardiac involvement, $50 \%$ of patients had depressed left ventricular function (left ventricular ejection fraction $<55 \%$ ) on echocardiography and myocardial edema suggestive of myocarditis on cMRI [26]. While short-term cardiac 
outcomes in these patients are reassuring, with almost all patients fully recovering at 2-week follow-up, there is some evidence that diastolic dysfunction may persist in a small subset of patients six months after acute illness [27]. Due to these severe findings, the American Academy of Pediatrics has recommended that patients with a diagnosis of MIS-C and cardiac involvement should not be cleared for physical activity for at least 3-6-month post-infection, and clearance should be obtained by a cardiologist [28].

There are three predominant hypotheses on the underlying pathophysiology of MIS-C. The first postulates a post-infectious autoimmune response. Patients with MIS-C present several weeks after acute infection, have low viral load, low immunoglobulin M (IgM), and high $\operatorname{IgG}$ and $\operatorname{IgA}$ expression [29]. It is therefore plausible that like other viral triggers of autoimmunity, SARS-CoV-2 activates an autoimmune response resulting in tissue damage [20,30]. A second theory is that there is an uncontrolled immune response to prolonged presence of SARS-CoV-2 antigen. Yonker et al. have shown that weeks after acute infection, SARS-CoV-2 antigen can still be found in the gastrointestinal tract of patients who developed MIS-C [31]. Finally, similar to patients with toxic shock syndrome, a third theory suggests "superantigen-like activity of the SARS-CoV-2 spike protein" $\rightarrow$ incites a dysregulated immune response with cytokine storm [30]. As it pertains to cardiovascular dysfunction, the release of cytokines (TNF- $a$ IL-1 $\beta$, IL-2, IL-6, and IFN- $\gamma$ ) is the known cause for left ventricular dysfunction, affecting both the extracellular matrix and myocyte contractility [32]. The pathophysiology of MIS-C may indeed be multifactorial, yet in order to tailor management, more research is needed to determine the downstream cardiac effects of this inflammation.

\section{Myopericarditis Post-mRNA COVID-19 Vaccination}

Myopericarditis post-mRNA COVID-19 vaccination in the adolescent and young adult population has recently been described [33-36]. It was not reported in the clinical vaccine trials likely due to the rarity of the complication and small sample size [33-36]. However, in June 2021, the CDC reported a post-vaccine myocarditis rate that was higher than expected from baseline. The myocarditis predominantly occurs in adolescent males after the second dose of the mRNA COVID-19 vaccine with the onset of chest pain ranging from one to five days after vaccine administration. As of November 24, 2021, the Vaccine Adverse Event Reporting System (VAERS) has received 1949 reports of myocarditis or myopericarditis after the mRNA COVID-19 vaccination among people 30 years of age and younger [37], with 1071 confirmed cases by the CDC and Food and Drug Administration [38]. One study showed the rate of myocarditis after the second dose of
Pfizer-BioNTech was approximately 54 cases per million doses administered to males aged 12-17 years [1]. The CDC estimates the incidence of myocarditis after the COVID-19 vaccine to be 1.2 per 100,000 doses administered to those between the ages of 18 and 29 years [3]. The Advisory Committee on Immunization Practices (ACIP) found the rate of myocarditis after the second dose of mRNA COVID-19 vaccine in the 12-29-year age group to be 40.6 cases per million in males and 4.2 cases per million in females [39]. In clinical trials, no cases of myocarditis have occurred in children aged five to 11 years who have received the COVID-19 vaccine [1].

Myocarditis involves inflammation of the myocardium, whereas myopericarditis involves the pericardium as well. Diagnosis is made clinically along with electrocardiogram (EKG), cardiac markers, echocardiogram, and cMRI to support the diagnosis. Typically, patients present with chest pain, shortness of breath, or palpitations within 1-5 days of receiving the second dose of the mRNA COVID-19 vaccine. While pathophysiology of COVID-19 vaccine-related myocarditis and myopericarditis is still unclear, there are few potential mechanisms proposed. COVID-19 vaccination may trigger an immune-mediated adverse response in those with genetically predisposed individuals with preexisting dysregulated pathways [40]. Another theory is that those with elevated heart-reactive autoantibodies, which are found in patients susceptible to myocarditis, may attack the cardiac myocytes after vaccination [41]. Diagnostic findings to suggest post-vaccine myocarditis or myopericarditis may include elevated troponin, elevated brain natriuretic peptide (BNP), elevated inflammatory markers, diffuse ST-segment elevation, nonspecific ST changes, T-wave inversions on EKG, left ventricular systolic dysfunction on echocardiography, left ventricular systolic dysfunction, and myocardial edema \pm late gadolinium enhancement based on T2-weighted imaging or T2 mapping on cMRI. Acute management is the same as for viral myocarditis/myopericarditis: supportive care to treat the symptoms, ensure adequate perfusion, and treat arrhythmias. While controversial, patients can receive non-steroidal anti-inflammatory drugs, immune globulin (IVIG), and steroids. Once patients have clinically improved and labs are downtrending, they are discharged home. Reported cases to date have shown that patients have a benign clinical course and favorable short-term outcomes. In most patients, follow-up 3-6 months after have shown resolution of symptoms with normal ventricular function [42]. No deaths from myocarditis post-mRNA COVID19 vaccination have been reported in the USA, with very rare deaths reported worldwide. A systematic international review of mortality following COVID-19 vaccination found two autopsy-confirmed cases of myocarditis causing death, one in a 22-year-old man following the Pfizer COVID-19 vaccine and one in a 42-year-old man following the Moderna 
vaccine [43]. The pathophysiology and long-term complications, if any, need to be studied further. Despite the risks of myocarditis or myopericarditis associated with vaccination, the benefits of vaccination likely outweigh the risk of COVID-19.

\section{Conclusion}

This review article serves to compile data regarding the cardiac complications associated with acute COVID-19 infection, MIS-C, and the mRNA COVID-19 vaccine in the pediatric population. Children who contract SARS-CoV-2 generally do well with either mild or no symptoms, but there is a small subset who have severe outcomes, with cardiac complications being one of the most significant. To prevent rare but serious outcomes of COVID-19 in children, to protect those who are not eligible for vaccination and those at high risk for severe disease, and to slow the spread of the virus in the community, the recommendation is widespread COVID-19 vaccination for those 5 years and older.

A September 2021 study in Israel analyzed risk ratios for mRNA vaccine-related myocarditis compared to a matched population of unvaccinated individuals aged 16 and above [44]. The investigators performed a separate and similar analysis of myocarditis risk in SARS-CoV-2-infected individuals matched with uninfected individuals. Although the mRNA COVID-19 vaccination was associated with an elevated risk for myocarditis with a risk ratio of 3.24, the same study found that acute infection with SARS-CoV-2 increased the risk for myocarditis with a risk ratio of 18.28. The risk for mRNA COVID-19 vaccine-related myocarditis was around 5 in 100,000, whereas myocarditis associated with acute COVID-19 was around 11 per 100,000 cases. The caveat is that this study focuses on individuals 16 and older and may not accurately represent the risks for young children. What remains true across all age groups is that SARS-CoV-2 is associated with a number of other cardiac and non-cardiac complications that have not been seen with the COVID-19 vaccination.

A study by Jain et al. [42] compared patients ranging in age from 12 to 20 years old who had COVID-19 vaccinerelated myocarditis to those who were hospitalized for MISC. They found that vaccine-related myocarditis is clinically distinct from MIS-C, is less severe with a milder presentation and hospital course, and is not associated with cardiac dysfunction requiring advanced support as compared to those patients with MIS-C.

The CDC has analyzed the risk of myocarditis after the mRNA COVID-19 vaccine and benefits of reduction of COVID-19-related hospitalization and death for each age group [3, 8]. Focusing on the 12-17-year age group which is the group most affected by vaccine-related myocarditis, for every million second-dose mRNA COVID-19 vaccinations, there is a risk of 8-10 myocarditis cases in females and 56-69 myocarditis cases in males. For this same age group and same number of vaccinations administered, the predicted benefit is prevention of 14,200 COVID-19 cases, 398 hospitalizations, 109 ICU admissions, and three deaths in females and males combined [3]. The risk-benefit analysis is overwhelmingly favorable for COVID-19 vaccination for those aged 12 and above [8].

In individuals 5-11, there have not been reported cases of vaccine-related myocarditis and the risks for severe COVID-19 disease and hospitalization are low. In Canada, the hospitalization rate in children aged 5-11 years with confirmed COVID-19 is $<0.3 \%$ and death is $<0.002 \%$ [45]. Although severe disease and death is low, this age group has been impacted by the COVID-19 pandemic in other ways, with virtual schooling, limited social interactions, and restricted and canceled extracurricular activities affecting mental and physical well-being [45]. Children who are vaccinated may have the benefits of less school disruptions, improved access to academic and extracurricular resources, and eased restrictions on social gatherings [45]. The benefits of vaccination in children are not limited to reducing medical complications from COVID-19, but also lessening the adverse effects from social distancing and isolation, preventing community spread, and protecting vulnerable and high-risk populations.

Multiple studies around the globe have shown that a two-dose mRNA COVID-19 vaccination series offers considerable protection against severe disease, hospitalization, and death caused by COVID-19 [46]. The CDC has reported an increased risk of myocarditis in the adolescent and young adult population after the mRNA COVID-19 vaccine, but this is a rare complication with a good prognosis. It is important to keep this risk in perspective, especially in the context of an ongoing pandemic with new SARS-CoV-2 variants of concern. Children, adolescents, and young adults are not immune to some of the complications associated with severe COVID-19 disease, and widespread vaccination is effective in mitigating severe medical complications, preventing further community spread, and lessening the disruptions associated with the pandemic, with the ultimate goal of ending this global crisis.

Author contributions Dr SEC drafted the initial manuscript and reviewed and revised the manuscript. Drs SMB, AC, Ghersin, and HSK each contributed to the manuscript and reviewed and revised the manuscript. All authors approved the final manuscript as submitted and agreed to be accountable for all aspects of the work.

Funding No funding/support for this review article. 


\section{Declarations}

Conflict of interest None.

\section{References}

1. Centers for Disease Control and Prevention. COVID-19 Vaccines for Children and Teens, updated November 23, 2021. Accessed 24 Dec 21. https://www.cdc.gov/coronavirus/2019ncov/vaccines/recommendations/children-teens.html?s_cid= 11369:cdc\%20children\%20covid\%20vaccine:sem.ga:p:RG:GM: gen:PTN:FY21

2. American Academy of Pediatrics. Children and COVID-19 Vaccination Trends. AAP analysis of data posted by the centers for disease control and prevention as of December 1, 2021. https:// downloads.aap.org/AAP/PDF/Child\%20Vaccinations\%20Rep ort\%20US\%20and\%20by\%20State\%20Dec\%201\%20revised.pdf

3. Wallace M, Oliver S (2021) COVID-19 mRNA vaccines in adolescents and young adults: Benefit-risk discussion. Presented at the Advisory Committee on Immunization Practice Meeting June 23, 2021 https://www.cdc.gov/vaccines/acip/meetings/ downloads/slides-2021-06/05-COVID-Wallace-508.pdf

4. Centers for Disease Control and Prevention. Provisional COVID-19 deaths by sex and age. Accessed 28 Nov 21. https:// data.cdc.gov/widgets/9bhg-hcku?mobile_redirect=true

5. Inciardi RM, Lupi L, Zaccone G et al (2020) Cardiac involvement in a patient with coronavirus disease 2019 (COVID-19). JAMA Cardiol 20:20. https://doi.org/10.1001/jamacardio.2020. 1096

6. Yu CM, Wong RS, Wu EB et al (2006) Cardiovascular complications of severe acute respiratory syndrome. Postgrad Med J $82: 140-144$

7. Alhogbani T (2016) Acute myocarditis associated with novel Middle East respiratory syndrome coronavirus. Ann Saudi Med 36:78-80

8. Bozkurt B, Kamat I, Hotez P (2021) Myocarditis with COVID19 mRNA vaccines. Circulation 144:471-484. https://doi.org/ 10.1161/CIRCULATIONAHA.121.056135

9. Shi S, Qin M, Shen B et al (2020) Association of cardiac injury with mortality in hospitalized patients with COVID-19 in Wuhan, China. JAMA Cardiol 323:1061-1069

10. Long B, Brady WJ, Koyfman A et al (2020) Cardiovascular complications in COVID-19. Am J Emerg Med S0735-6757(20):30277-30281

11. Lara D, Young T, Del Toro K, Chan V, Ianiro C, Hunt K, Kleinmahon J (2020) Acute fulminant myocarditis in a pediatric patient With COVID-19 infection. Pediatrics 146(2):e20201509. https://doi.org/10.1542/peds.2020-1509

12 Trogen B, Gonzalez FJ, Shust GF (2020) COVID-19-associated myocarditis in an adolescent. Pediatric Infect Dis J 39(8):e204e205. https://doi.org/10.1097/INF.0000000000002788

13. Gnecchi M, Moretti F, Bassi EM, Leonardi S, Totaro R, Perotti L, Zuccaro V, Perlini S, Preda L, Baldanti F, Bruno R, Visconti LO (2020) Myocarditis in a 16-year-old boy positive for SARSCoV-2. Lancet. https://doi.org/10.1016/S0140-6736(20)31307-6

14. Santi AD, Aquino P, Dorfman M (2021) Atrial fibrillation in a child with COVID-19 infection. Cardiol Young 31(2):318-321. https://doi.org/10.1017/S1047951120003893

15. Hopkins KA, Webster G (2021) Aberrated supraventricular tachycardia associated with neonatal fever and COVID-19 infection. BMJ Case Rep CP 14:e241846

16. Clark DE, Parikh A, Dendy JM, Diamond AB, George-Durrett K, Fish FA, Slaughter JC, Fitch W, Hughes SG, Soslow JH
(2021) COVID-19 myocardial pathology evaluation in athletes with cardiac magnetic resonance (COMPETE CMR). Circulation 143:609-612. https://doi.org/10.1161/CIRCULATIO NAHA. 120.052573

17. Boehmer TK, Kompaniyets L, Lavery AM et al (2021) Association between COVID-19 and myocarditis using hospital-based administrative data-United States, March 2020-January 2021. MMWR Morb Mortal Wkly Rep 70:1228-1232. https://doi.org/ 10.15585/mmwr.mm7035e5

18. Children and Covid-19: State-Level Data Report. Home. www. aap.org/en/pages/2019-novel-coronavirus-covid-19-infections/ children-and-covid-19-state-level-data-report/

19. CDC Covid Data Tracker. Centers for Disease Control and Prevention, Centers for Disease Control and Prevention, covid.cdc. gov/covid-data-tracker/\#mis-national-surveillance

20. Bohn MK et al (2021) Multi-inflammatory syndrome in children: a view into immune pathogenesis from a laboratory perspective. J Appl Lab Med. www.ncbi.nlm.nih.gov/pmc/articles/ PMC8499820/

21. Duarte-Salles T, Vizcaya D, Pistillo A, Casajust P, Sena AG, Lai LYH et al (2021) Thirty-day outcomes of children and adolescents with COVID-19: an international experience. Pediatrics. https://doi.org/10.1542/peds.2020-042929

22. Rafferty MS et al (2021) Multisystem inflammatory syndrome in children (Mis-C) and the coronavirus pandemic: current knowledge and implications for public health. J Infect Public Health. www.sciencedirect.com/science/article/pii/S18760341210001 25

23. Dufort EM et al (2020) Multisystem inflammatory syndrome in children in New York State. NEJM 10:20. https://doi.org/10.1056/ NEJMoa2021756

24. Belot A et al (2020) SARS-COV-2-related paediatric inflammatory multisystem syndrome, an epidemiological study, France, 1 March to 17 May 2020. Eurosurveillance, European Centre for Disease Prevention and Control, 4 June 2020, www.eurosurvei llance.org/content. https://doi.org/10.2807/1560-7917.ES.2020. 25.22.2001010

25. Son MBF et al (2021) Multisystem inflammatory syndrome in children-initial therapy and outcomes. NEJM. https://doi.org/ 10.1056/nejmoa2102605

26. Theocharis P et al (2021) Multimodality cardiac evaluation in children and young adults with multisystem inflammation associated with Covid-19. Eur Heart J Cardiovasc Imaging 22(8):896-903

27. Capone CA, Misra N, Ganigara M, Epstein S, Rajan S, Acharya SS, Hayes DA, Kearney MB, Romano A, Friedman RA, Blaufox AD, Cooper R, Schleien C, Mitchell E (2021) Six month followup of patients with multisystem inflammatory syndrome in children. Pediatrics 148(4):e2021050973. https://doi.org/10.1542/ peds.2021-050973

28. Trisha K (2021) AAP updates guidance on when youths with Covid-19 can return to physical activity. Am Acad Pediatr. publications.aap.org/aapnews/news/7110?autologincheck $=$ redirected

29. Gruber CN, Patel RS, Trachtman R, Lepow L, Amanat F, Krammer F, Wilson KM, Onel K, Geanon D, Tuballes K, Patel M, Mouskas K, O’Donnell T, Merritt E, Simons NW, Barcessat V, Del Valle DM, Udondem S, Kang G et al (2020) Mapping systemic inflammation and antibody responses in multisystem inflammatory syndrome in children (mis-C). Cell 183(4):15. https://doi. org/10.1016/j.cell.2020.09.034

30. Mazer MB, Bulut Y, Brodsky NN, Lam FW, Sturgill JL, Miles SM, Shein SL, Carroll CL, Remy KE (2022) Multisystem inflammatory syndrome in children. Pediatr Crit Care Med. https://doi. org/10.1097/pcc.0000000000002897

31. Yonker LM et al (2021) Multisystem inflammatory syndrome in children is driven by zonulin-dependent loss of gut mucosal barrier. J Clin Investig. https://doi.org/10.1172/JCI149633 
32. Prabhu SD et al (2004) Cytokine-induced modulation of cardiac function. Circ Res 95:1140-1153. https://doi.org/10.1161/01.res. 0000150734.79804.92

33. Marshall M, Ferguson ID, Lewis P et al (2021) Symptomatic acute myocarditis in 7 adolescents after Pfizer-BioNTech COVID19 vaccination. Pediatrics 148(3):e2021052478

34. Dionne et al (2021) Association of myocarditis with BNT162b2 messenger RNA COVID-19 vaccine in a case series of children. JAMA Cardiol. https://doi.org/10.1001/jamacardio.2021.3471

35. Shimabukuro T (2021) COVID-19 vaccine safety updates. https:// www.fda.gov/media/150054/download.jamacardio.2021.2821

36 Shay DK, Shimabukuro TT, DeStefano F (2021) Myocarditis occurring after immunization with mRNA-based COVID-19 vaccines. JAMA Cardiol. https://doi.org/10.1001/Jamacardio.2021. 2821

37. Centers for Disease Control and Prevention. The vaccine adverse event reporting system (VAERS) results. Accessed 2 Dec 2021. https://wonder.cdc.gov/vaers.html

38. Centers for Disease Control and Prevention. Selected Adverse Events Reported after COVID-19 Vaccination. Accessed 2 Dec 2021. https://www.cdc.gov/coronavirus/2019-ncov/vaccines/ safety/adverse-events.html

39 Gargano JW, Wallace M, Hadler SC et al (2021) Use of mRNA COVID-19 vaccine after reports of myocarditis among vaccine recipients: update from the advisory committee on immunization practices-United States, June 2021. MMWR Morb Mortal Wkly Rep 70:977-982. https://doi.org/10.15585/mmwr.mm7027e2

40. Caso F, Costa L, Ruscitti P, Navarini L, Del Puente A, Giacomelli R, Scarpa R (2020) Could Sars-coronavirus-2 trigger autoimmune and/or autoinflammatory mechanisms in genetically predisposed subjects? Autoimmun Rev 19:102524. https://doi.org/10.1016/j. autrev.2020.102524

41. Caforio AL, Mahon NJ, Tona F, McKenna WJ (2002) Circulating cardiac autoantibodies in dilated cardiomyopathy and myocarditis: pathogenetic and clinical significance. Eur J Heart Fail 4:411-417. https://doi.org/10.1016/s1388-9842(02)00010-7

42. Jain et al (2021) COVID-19 vaccination-associated myocarditis in adolescents. Pediatrics 148(5):e2021053427

43 Sessa F, Salerno M, Esposito M, Di Nunno N, Zamboni P, Pomara C (2021) Autopsy findings and causality relationship between death and COVID-19 vaccination: a systematic review. J Clin Med 10(24):5876. https://doi.org/10.3390/jcm10245876

44 Barda N, Dagan N, Ben-Shlomo Y et al (2021) Safety of the BNT162b2 mRNA COVID-19 vaccine in a nationwide setting. N Engl J Med. https://doi.org/10.1056/NEJMoa2110475

45. An Advisory Committee Statement (ACS) National Advisory Committee on Immunization (NACI) Recommendation on the use of the Pfizer-BioNTech COVID-19 vaccine $(10 \mathrm{mcg})$ in children 5-11 years of age. Public Health Agency of Canada. Published November 19, 2021. https://www.canada.ca/content/dam/phacaspc/documents/services/immunization/national-advisory-commi ttee-on-immunization-naci/recommendations-use-covid-19-vacci nes/pfizer-biontech-10-mcg-children-5-11-years-age/pfizer-biont ech-10-mcg-children-5-11-years-age.pdf?fbclid=IwAR1CWbDn kGxg-lwdfEB6Mu4YMJ1zubmRFP_fCyiIESeV3KcIcJ_131tYj50

46. Centers for Disease Control and Prevention. Science brief: COVID-19 vaccines and vaccination updated Sept 15, 2021. Accessed 20 Nov 21. https://www.cdc.gov/coronavirus/2019ncov/science/science-briefs/fully-vaccinated-people.html

Publisher's Note Springer Nature remains neutral with regard to jurisdictional claims in published maps and institutional affiliations. 